\title{
SADIN, E. LA HUMANIDAD AUMENTADA: LA ADMINISTRACIÓN DIGITAL DEL MUNDO. 160 PÁGS. BUENOS AIRES, 2018: EDITORIAL CAJA NEGRA
}

Roberto Pizarro Contreras ${ }^{1}$

Universidad de Chile, Chile

Evocando la "personalidad" de la supercomputadora HAL 9000 de la película 2001: Odisea en el Espacio, que funge como metáfora para su planteamiento, el ensayista y filósofo francés Éric Sadin, lleva a cabo en La humanidad aumentada: La administración digital del mundo, Premio Hub al ensayo más influyente sobre lo digital, una genealogía y caracterización de la técnica contemporánea, y en particular de la inteligencia de la técnica.

La aumentación a la que se refiere el título de la obra señala, por un lado, la naturaleza que tendría la inteligencia de la técnica, pues lejos de la perspectiva originaria que concebía antropomórficamente a la inteligencia artificial (IA) -hoy su variante más representativa -, aquella ostenta una estructura altamente fragmentada (se realiza en la sinergia de pequeñas unidades que operan en dominios bien acotados de la realidad) y su impacto, sin embargo, afecta globalmente a los seres y las cosas (p. 21). (Asimilada neurobiológicamente, en contraste, la inteligencia humana está basada en redes neuronales orgánicas y aun un grupo de humanos con la más vasta influencia, en ausencia del recurso tecnológico, probablemente nunca conseguiría equiparar la precisión y velocidad inferenciales de su pretendida sustituta.)

Una expresión tecnológica de este tipo de inteligencia desperdigada lo constituyen un sensor de temperatura y una cámara de reconocimiento biométrico, los cuales podrían componer, mediante un software que procese la data recopilada por ambos dispositivos, un sistema inteligente de control del confort en un entorno de trabajo, correlacionando las expresiones faciales capturadas por el lente de la cámara con la temperatura ambiental. No hay que olvidar en todo caso, asevera Sadin, que el tipo técnico de intuición está basado en "percepciones colectivas e individuales basadas sin cesar en procesos estimativos fácticos [matemático-binarios] incapaces de aprehender plenamente la verdad multiestratificada de nuestras realidades" ( $p$. 119); lo que consiguen aprehender, por lo demás, lo hacen con un alcance tal que excede "monstruosamente" nuestros límites humanos.

Por otro lado, con "aumentación" el autor pretende poner de manifiesto la posibilidad de que, en el marco de una fusión entre humanos y máquinas, a la que se refiere como "antrobología" (una combinación de los términos ánthrōpos,

\footnotetext{
${ }^{1}$ Magíster en Filosofía. Universidad de Chile. E-mail: rpizarroc@hotmail.com
} 
robot y logos), el ser humano viera modificada su intelección (en el sentido de razonar y conocer). ¿Llegaremos a ser unos cyborgs que, en lugar de reflexionar si alguien se siente o no cómodo con nosotros, si alguien nos ama o no, calcularemos automáticamente el estado emocional de toda la audiencia en derredor? De ser esto posible, la preocupación en ese sentido, el amor, ya no tendrían cabida como problemas o aspectos profundos de una futura "humanidad. Su solución vendría dada por defecto.

En cuanto a esto, conviene aclarar igualmente que el asunto en cuestión no es determinar una potencial utilidad, o bien, la bondad o perjuicio de la sujeción de la inteligencia humana a la inteligencia de la técnica. Coincidiendo con Heidegger en su Pregunta por la técnica, para el intelectual francés aquello no sería estar a la altura del problema. Más bien de lo que se trata es de que, en la disolución indefectible que experimenta el sujeto moderno (p. 30), comprendamos los múltiples retos que nos plantea hoy el advenimiento de una técnica inteligente, un "retorno de HAL", y su propensión a administrar la realidad que humanos y máquinas comparten (p. 35). De ahí que una antrobología tenga asimismo como base filosófica una fenomenología tecnológica contemporánea, que haga posible un estado de vigilia continua respecto de las innovaciones industriales, así como una atención a los usos individuales y a las prácticas sociales en perpetua reconstitución (p. 32). "Es el imperativo de desplegar la potencia crítica para posicionarse conscientemente respecto de la verdad impuesta por la técnica." (pp. 154-155).

En la dimensión genealógica del ensayo, la invención del smartphone marca el fin de la revolución digital -y el inicio, en consecuencia, de la transformación digital del mundo -, representando "el primer objeto que generalizará, a largo plazo, el fenómeno de la realidad aumentada" (p. 57). Esta aumentación, en lo que atañe a nuestra humanidad, no es descartable como la del walkman portable, ya que entonces ha acontecido una conversión digital de numerosos segmentos de la realidad, lo que la torna indispensable, proyectando una nueva era que reúne los requisitos de una fusión anunciada entre cuerpos orgánicos y artificiales (p. 58), la instauración propiamente dicha de una condición mixta -humano/artificial-, de una antrobología (p. 61).

Esta configuración genera formas inéditas de existencia y redefine las relaciones históricas con el espacio y el tiempo, de los que sabemos, desde Kant, que estructuran la base de nuestra experiencia. Es una vida que, hasta el momento, fue llevada adelante por elecciones inciertas inspiradas en una sensibilidad limitada a capacidades de aprehensión relativas, que se ve "aumentada" o curvada por procesos cognitivos en parte superiores y más avezados que los nuestros. (p. 60).

En la dimensión de caracterización, el libro propone una serie de hechos cruciales que marcan la condición antrobológica, destinada a estatuirse como conclusión de 
la obra, y que al mismo tiempo permiten entender los atributos principales de la inteligencia de la técnica. Ellos son:

- La duplicación digital del mundo y su hipervigilancia, que señala una hiperconexión multiarquitectura y multiplataforma de la técnica a los entes del mundo (p. 113), propiciada por la miniaturización y extensión sensible por medio de sensores de la primera, y que queda bien representada por una de las dimensiones clave de la transformación digital, a saber: el Internet de las Cosas (IoT, por sus siglas en inglés). La reducción numeraria de los seres y las cosas es lo que hace posible su computación y es esta la base para una suerte de clarividencia de la técnica, que a cada instante tiende a la omnisciencia (pp. 77-88), y para el ejercicio demiúrgico del control.

- La adaptabilidad del código legitima el error en la predicción computacional y su corrección en tiempo real como fundamento de la algoritmia: el código evoluciona instantánea y eternamente (p. 116). Esta capacidad mienta a su vez otra, la de correlación multivariable y que Sadin describe como un develar técnico capaz de percibir, tal como hace el Big Data, con mayor precisión la naturaleza compleja, ciertos aspectos y vínculos insospechados y decisivos, de algunas de nuestras acciones y, en general, de nuestras realidades (p. 65).

- La delegación progresiva de poder a los procesadores, por su lado, dice relación con la capacidad de toma de decisión transferida a la técnica y que da sus primeros pasos con la aparición de los sistemas expertos en los años 90’ (p. 63). Es representativo de esta disposición de la técnica la facultad de sugerencia de los distintos aplicativos de los smartphones (por ejemplo, aquellos que nos prescriben cuándo nuestra quema de calorías ha alcanzado su meta diaria tras la detección de cierto kilometraje recorrido).

- La afección o humanización sistémicas contempla la potestad de la inteligencia de la técnica de "humanizar" sus apéndices "en razón" de reacciones emocionales, por una parte, $y$, por otra, de dirimir dilemas éticos (pp. 119-122). Esto incrementa otro potencial suyo previo y fundamental, esto es, su infiltración solapada (soft) sobre lo real.

- Por último, la totemización o sacralización de los productos tecnológicos (pp. 89-106), que, junto a la humanización antes expuesta, acaba por naturalizar el progreso técnico y persuade a la humanidad sobre lo inocuo y necesario que es para llevar a su culminación la condición de aquella.

Cuanto se ha dicho, es decir, la capacidad in crescendo de actuación y de respuesta controladoras de la inteligencia de la técnica, que pueden resumirse en la asistencia (o control) hiperindividualizada (p. 82), determinan en última instancia el 
surgimiento de un orden artificial o gubernamentalidad algoritmica que implica simultáneamente un proceso de civilización computarizada (pp. 137-141) que no es sino la emergencia de una humanidad que se mueve y piensa electrónicamente al son de los comandos computacionales. Es así que "la pregunta kantiana '¿Qué puedo conocer?', que funda la epistemología moderna, debe ser revisada desde cero en virtud de la aparición de un nuevo entorno cognitivo" (p. 145). Se trata, en síntesis, de la producción de una nueva metafísica de la experiencia que lejos de observar los "objetos" a la distancia, los torna accesibles al sujeto de una manera que solo existe aquello que es codificado. Las consecuencias están por verse aun: aguardamos "una nueva era compleja de la humanidad o se anuncia nuestra absorción en la matriz omnisciente que nosotros mismos hemos creado" (p. 35).

Con todo, se insistirá en que a pesar del carácter ominoso que pudiera revestir la genealogía y caracterización del imperio inteligente erigido por la técnica contemporánea, frente a su presencia e influencia insoslayables, se nos convoca no a mirar con ojos amenazantes ni mucho menos con resignación, sino a una asunción lúcida y responsable de nuestra condición, porque solo en este autorrepliegue será posible atisbar alternativas para el descentramiento del humanismo moderno, cuando menos "marcar un distanciamiento de la figura de HAL, ya no para neutralizarla o aniquilarla, sino para instaurar un juego vital abierto y dinámico" (p. 155). 